\title{
Fortifications et habitats de hauteur protohistoriques en Alsace Bilan des recherches récentes
}

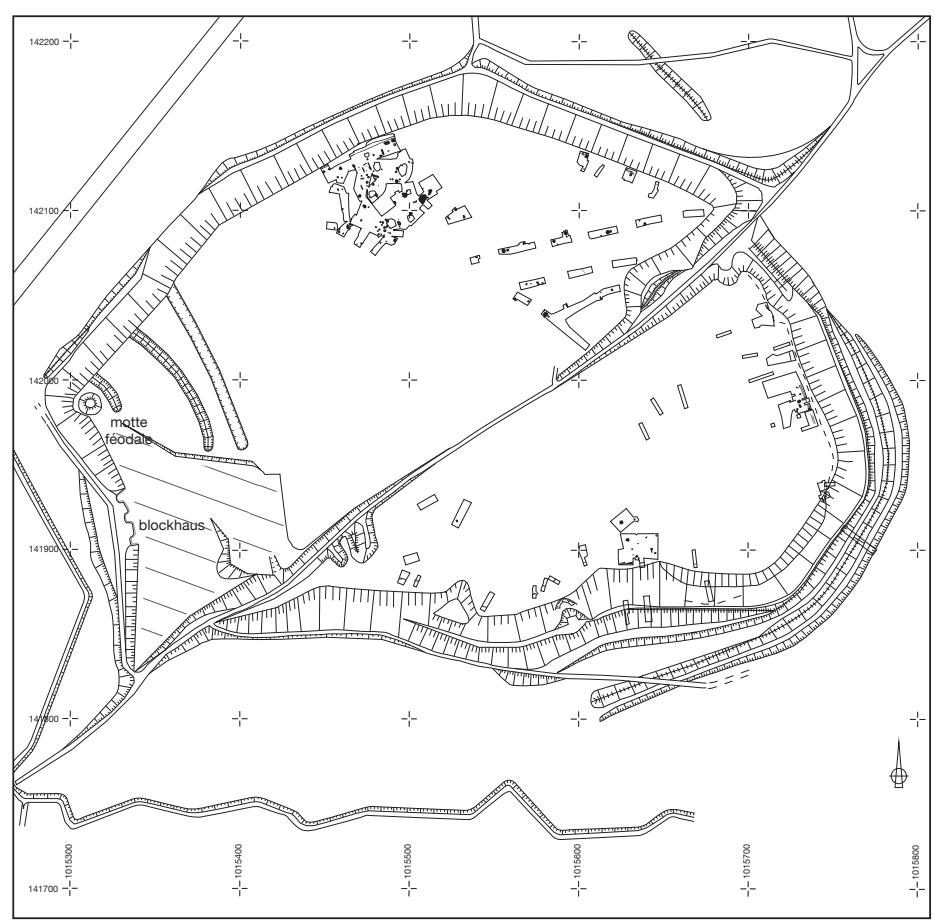

Résumé:

Le développement récent de programmes de recherche sur les fortifications protohistoriques d'Alsace incite à proposer un bilan des connaissances disponible sur ces sites. Plusieurs aspects ont été abordés : les données morphologiques, topographiques ou architecturales ont été passées en revue avant qu'une analyse chronologique des occupations fortifiées ne soit effectuée. En définitive, une vision renouvelée des habitats de hauteur peut être proposée.

\section{Mots-clés :}

Fortifications, habitat de hauteur, âge du Bronze, âge du Fer, rempart, architecture

Abstract:

The recent development of research on the protohistoric fortifications in Alsace encourages to propose an assessment of the knowledge available on these sites. Several aspects were broached: morphological, topographical or architectural data were reviewed before a chronological analysis of the fortified settlements was carried out. Ultimately, a renewed vision of hillforts can be proposed.

Keywords:

Fortifications, Hillforts, Bronze Age, Iron Age, Rempart, Architecture 


\section{Recherches anciennes et dynamiques nouvelles}

Les sites de hauteur d'Alsace ont assez tôt attiré l'attention des érudits et historiens locaux qui en détaillent les vestiges et cherchent à en définir l'origine et la fonction. Dès 1583, dans son Architectura von Vestungen, D. Specklin, ingénieur militaire, propose les premières descriptions de fortifications vosgiennes, des châteaux pour l'essentiel. Près de deux siècles plus tard, J.-B. Schoepflin publie la première étude d'envergure consacrée à l'histoire générale de l'Alsace, l'Alsatia Illustrata (1751-1761), dans laquelle il attribue nombre d'enceintes, dont le «Mur Païen » du Mont Sainte-Odile, à l'époque romaine. Cet ouvrage, traduit en 1849 (Schoepflin, Ravenez 1849-1852), restera la base sur laquelle s'appuieront, durant tout le XIX ${ }^{\mathrm{e}}$ s., de nombreuses recherches qui reprendront cette hypothèse.

La première moitié du siècle voit quelques chercheurs se concentrer plus précisément sur les enceintes fortifiées, qui accèdent ainsi au statut d'objets archéologiques. En 1823, P. de Golbéry dans son Mémoire sur quelques anciennes fortifications des Vosges, ou J. Schneider en 1844 avec les Beiträge zur Geschichte der alten Befestigungen in den Vogesen, proposent de véritables études historiques fondées sur l'analyse des sites de hauteur. Suivant en cela un mouvement beaucoup plus large, cette dynamique aboutira à la publication, entre la fin du XIX ${ }^{\mathrm{e}}$ et le début du $\mathrm{XX}^{\mathrm{e}}$ s., de plusieurs inventaires, généralement adossés à des explorations de terrain : les travaux de G. Bleicher et F. Faudel pour le territoire alsacien (Bleicher, Faudel 1880), de K.-S. Gutmann pour la Haute-Alsace (Gutmann 1913), ou encore de C. Mehlis pour les Vosges du Nord et le Palatinat (Mehlis 1900), offrent une vision élargie des sites dont la datation pré- et protohistorique est maintenant reconnue.

L'ensemble de ces travaux est synthétisé en 1926 par R. Forrer dans un article de référence, Des enceintes fortifiées préhistoriques, romaines et anhistoriques d'Alsace, publié sous les auspices de la Société pour la conservation des monuments historiques (Forrer 1926). Il y recense la plupart des sites alors connus, sans que toutefois certains manques, pourtant assez évidents, ne soient explicités. La documentation réunie est abondante : des plans et des coupes ou profils de remparts sont proposés pour la grande majorité des 173 enceintes dénombrées qui sont organisées selon une triple analyse, morphologique, chronologique et fonctionnelle. Cette étude marque la fin de plusieurs décennies de recherches sur les fortifications ; les fouilles se font moins nombreuses et seuls quelques rares articles sont publiés après cette date.

Si on excepte les travaux menés par les services du Landesamt für Ur- und Frühgeschichte entre 1940 et 1944 pour démontrer l'origine germanique de plusieurs fortifications, il faut attendre le milieu des années 1960 pour voir reprendre les fouilles sur les enceintes alsaciennes. De 1963 à 1972, H. Zumstein fouille le Mont Sainte-Odile et précise la chronologie des occupations du plateau du couvent ; le Britzgyberg fait l'objet de sondages répétés entre 1965 et 1989 par R. et J. Schweitzer ; enfin, la « station d'altitude » du Hohlandsberg est étudiée par C. Bonnet de 1965 à 1985 puis par J.-J. Wolf dans la décennie suivante. Les grandes synthèses suprarégionales (Roussel-Claerr 1983, Buchsenschutz 1984, ou Deyber 1984, entre autres) qui suivent ce regain d'intérêt pour les fortifications ne concernent pas spécifiquement l'Alsace; elles sont fondées sur des recensions bibliographiques qui font apparaître, en creux, mais de façon assez nette, le peu d'avancées significatives sur le sujet depuis 1926.

À partir du milieu des années 1990, prenant acte de ce constat (Fichtl et al. 1997), un groupe de chercheurs de l'université de Strasbourg et du Service Régional de l'Archéologie ouvre une série de fouilles sur des sites fortifiés : au Mont Sainte-Odile (1994-1995, S. Fichtl, A.-M. Adam), au Hexenberg à Leutenheim (1994-2007, A.-M. Adam puis M. Lasserre), au Maimont à Niedersteinbach (1996, S. Fichtl), au Fossé des Pandours au col de Saverne (1995-2006, S. Fichtl puis C. Féliu). À partir du milieu des années 2000, de nouveaux sites font l'objet de travaux de terrain : le Britzgyberg à Illfurth (depuis 2003, A.M. Adam), la Heidenstadt à Ernolsheim-lès-Saverne (20072010, C. Féliu), le Hohlandsberg à Wintzenheim (2010, F. Delrieu) ou le Kastelberg à Kœstlach (2011-2014, M. Landolt). Depuis 2012, un groupe de recherche s'est structuré dans le cadre de l'UMR7044 - Archimède de Strasbourg ; il s'est donné pour objectifs de préciser la nature, la fonction et la chronologie des fortifications de hauteur alsaciennes, dans un cadre diachronique large, de la Protohistoire au Moyen Âge (Féliu, Schwien 2016). D'importants travaux de prospection et de compilation de données anciennes et récentes ont été effectués ; ils ont abouti à la réalisation de deux masters, un pour chacun des départements alsaciens, qui offrent la possibilité de disposer de données de terrain fiables sur une grande majorité des fortifications de la région (Walter 2015 et Wittlin 
2016, d'où sont tirées la grande majorité des informations suivantes). Un troisième mémoire a été soutenu sur les fortifications du premier âge du Fer dans l'est de la Gaule (Robin 2016), un quatrième est en préparation sur l'architecture et la typologie des remparts protohistoriques entre Ardennes et Vosges (travaux d'A. Di Vincenzo). Dans le même temps, de nouvelles fouilles ont été initiées : au Frankenbourg à Neubois (depuis 2014, C. Féliu), au Schiefferberg à Oberhaslach (depuis 2016, S. Gentner et M. Walter), ou encore au Maimont à Niedersteinbach (depuis 2016, R. Wassong).

L'accroissement important du nombre des programmes de recherche, inventaires et travaux de terrain, depuis une quinzaine d'années donne l'opportunité de proposer une vision réactualisée des fortifications et sites de hauteur protohistoriques en Alsace.

\section{2. État de la documentation, données générales}

L'inventaire des enceintes de hauteur d'Alsace, qui compte environ 175 occurrences depuis les travaux de Forrer, peut être très nettement réduit pour ce qui concerne la Protohistoire. Dans le cadre de cet article, ne seront retenus que les sites effectivement occupés durant les âges des Métaux, ainsi que les installations non datées, les « enceintes anhistoriques » de la SPF. La découverte de l'un ou l'autre objet protohistorique isolé dans la cour d'un château ou à l'emplacement d'une enceinte médiévale, comme la hache à talon de l'âge du Bronze moyen mise au jour au Petit Ringelsberg à Oberhaslach (Walter 2015, n²7) par exemple, n'a pas été jugée suffisante pour intégrer ces sites à l'étude. Certaines enceintes ont également été écartées sur la base de critères relativement discrets, comme la présence d'un mur étroit à double parement qui ne saurait être intégré à l'ensemble des remparts protohistoriques dont l'architecture met en œuvre de grandes masses de matériaux et atteint des largeurs assez importantes; de même, certains tracés, très anguleux ou de très faible surface, comme les deux redoutes du col du Bonhomme (Wittlin 2016, n $^{\circ} 3$ et 4) qui semblent plutôt appartenir à des terrassements modernes, n'ont pas été considérés comme protohistoriques. Au contraire, quelques sites abondamment documentés anciennement, mais aujourd'hui disparus, comme le Hartmannswillerkopf à Hartmannswiller, ont été intégrés à l'étude. En définitive, sur la base des travaux de master mentionnés plus haut, un petit ensemble de 39 enceintes et sites de hauteur a été retenu pour l'Alsace (Fig. 1 et 2).

\subsection{Géographie et topographie des implantations}

Pour des raisons topographiques évidentes, la plupart des enceintes et sites de hauteur protohistoriques se situent dans le massif vosgien. Celles-ci se concentrent d'une part au niveau des Vosges du nord jusqu'au seuil de Saverne et à la vallée de la Bruche, et d'autre part au sud du massif, entre les vallées de la Fecht et de la Thur. La zone centrale ne semble que peu investie : seuls deux sites, le Mont Sainte-Odile et le Frankenbourg, y ont été reconnus. La plupart des sites vosgiens est installée sur le rebord du massif et surplombe, généralement de plusieurs centaines de mètres, la vallée du Rhin, bénéficiant ainsi de positions stratégiques indéniables. D'autres, moins nombreux, se situent en retrait, généralement dans des vallées secondaires. Les premiers contreforts du Jura et les collines du Sundgau sont également occupés par une petite série de fortifications. Enfin, il faut mentionner le site du Hexenberg à Leutenheim, installé au sommet d'une butte témoin détachée de la terrasse alluviale distante d'un kilomètre environ, qui domine la plaine rhénane d'une dizaine de mètres et occupe ainsi une position particulière en Alsace.

L'implantation de la grande majorité des enceintes s'appuie sur le relief. La moitié d'entres elles (21/39) est installée sur des éperons aux flancs plus ou moins abrupts. Le site de la Heidenstadt qui occupe un segment de crête bordée naturellement par des falaises au nord et au sud, et qui est délimité par deux remparts à l'est et à l'ouest peut être rapproché de cet ensemble dont la morphologie générale est étroitement liée à la topographie. Un tiers des sites retenus est installé sur des sommets parfois escarpés (14/39), auxquels on peut ajouter la butte témoin du Hexenberg. Enfin, seuls deux sites, Lembach et Mackwiller, semblent ne pas avoir été implantés sur une position spécifiquement avantageuse ; ils correspondent cependant l'un et l'autre à des mentions anciennes dont la validité n'est pas assurée.

Le tracé des remparts, pour la plupart de simples remparts de barrage, est assez cohérent avec les modèles généralement appliqués aux fortifications protohistoriques dont la défense est en grande partie assurée par la topographie. Quelques enceintes de contour ont toutefois été construites sur de sites naturellement défendus, au Fossé des Pandours, à Kœstlach ou au Britzgyberg. 


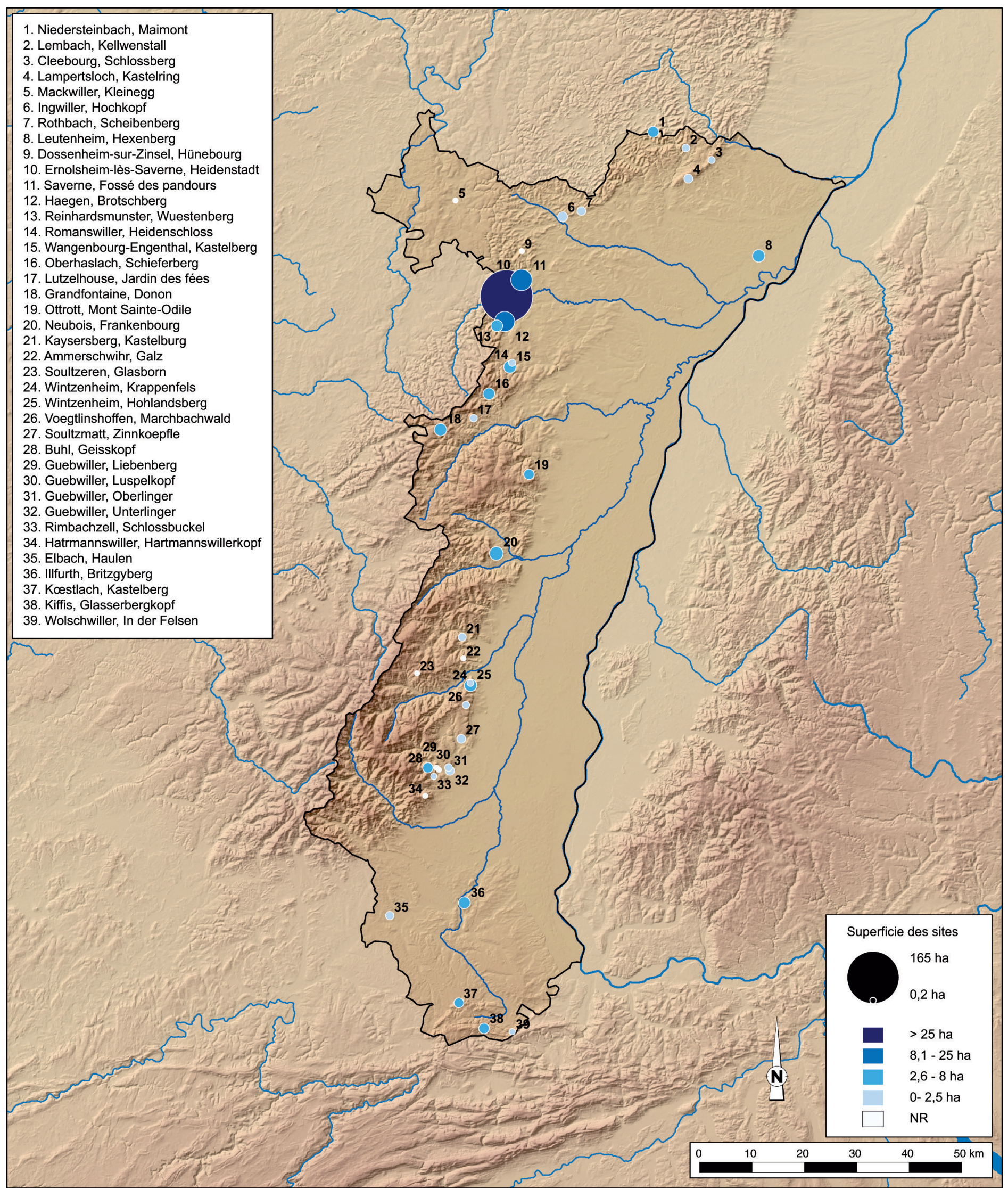

11 Carte de localisation des fortifications retenues pour l'étude - DAO : C. Féliu (fond : SRTM-NASA). 


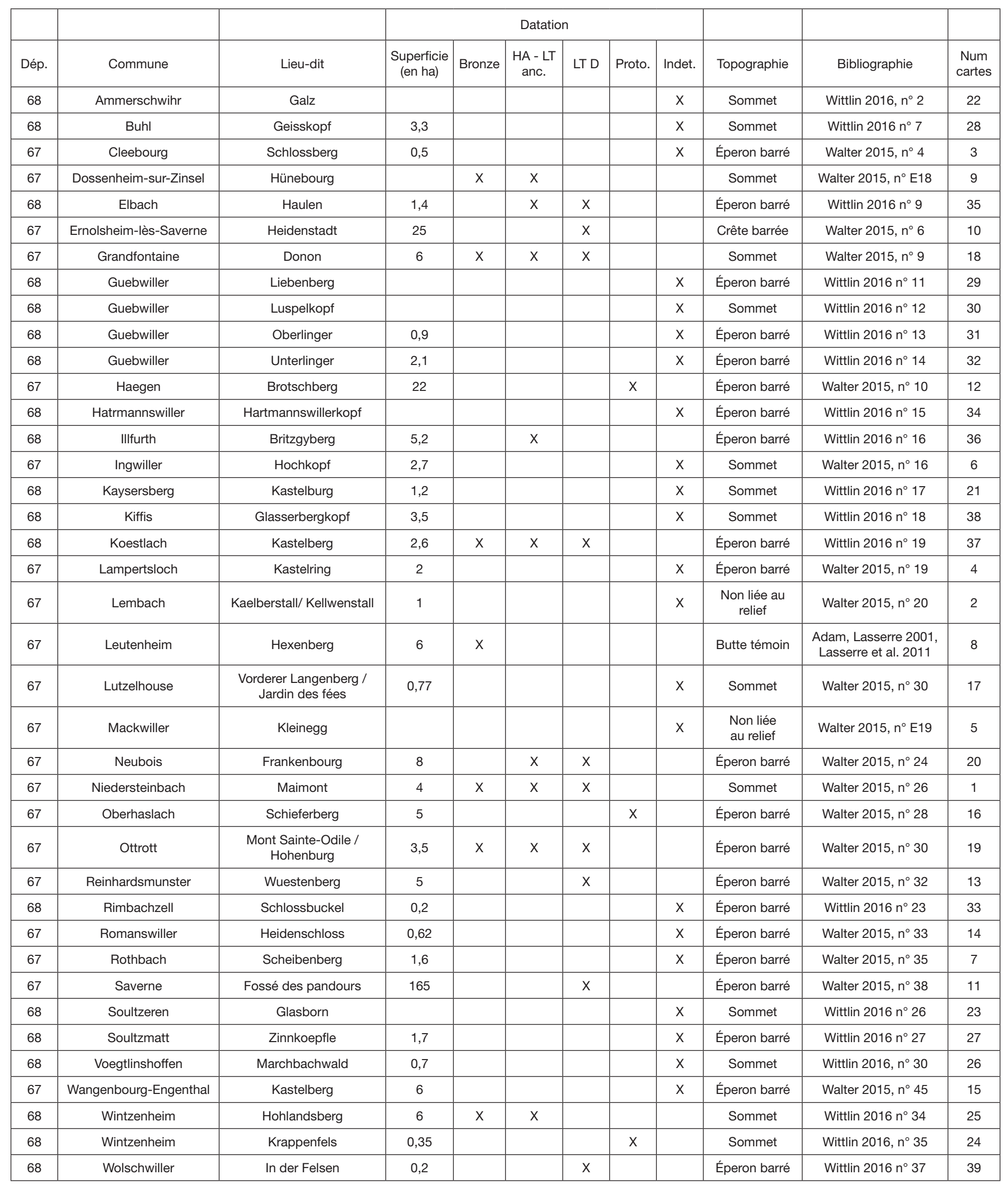

12 Inventaire des sites retenus pour l'étude - DAO : C. Féliu. 
Deux fortifications adoptent des implantations particulières qui méritent que l'on s'y attarde. Le Hohlandsberg à Wintzenheim est installé sur un sommet aux pentes assez raides. Le rempart continu de l'âge du Bronze, qui isole une superficie de 6 ha environ, n'est pas construit autour de ce point haut, selon un tracé parallèle aux courbes de niveaux, mais il est implanté dans la pente et délimite un espace grossièrement rectangulaire sur le versant oriental de l'éminence. Au Frankenbourg à Neubois, la configuration est quelque peu différente mais obéit à des considérations identiques : le promontoire du Schlossberg sur lequel est installé le site est entouré de pentes relativement raides et n'est accessible qu'à l'ouest, par un petit col. Le rempart de La Tène finale ne semble pas se développer sur le pourtour du site mais plutôt se restreindre à la zone d'accès la plus aisée, tout comme l'occupation protohistorique que les prospections cantonnent sur le versant ouest du sommet, sur une surface d'environ 4 ha.

Les superficies encloses, immédiatement accessibles à l'étude pour 32 des 39 fortifications, varient de 0,2 ha à 165 ha (Fig. 3). Trois sites se distinguent assez nettement des

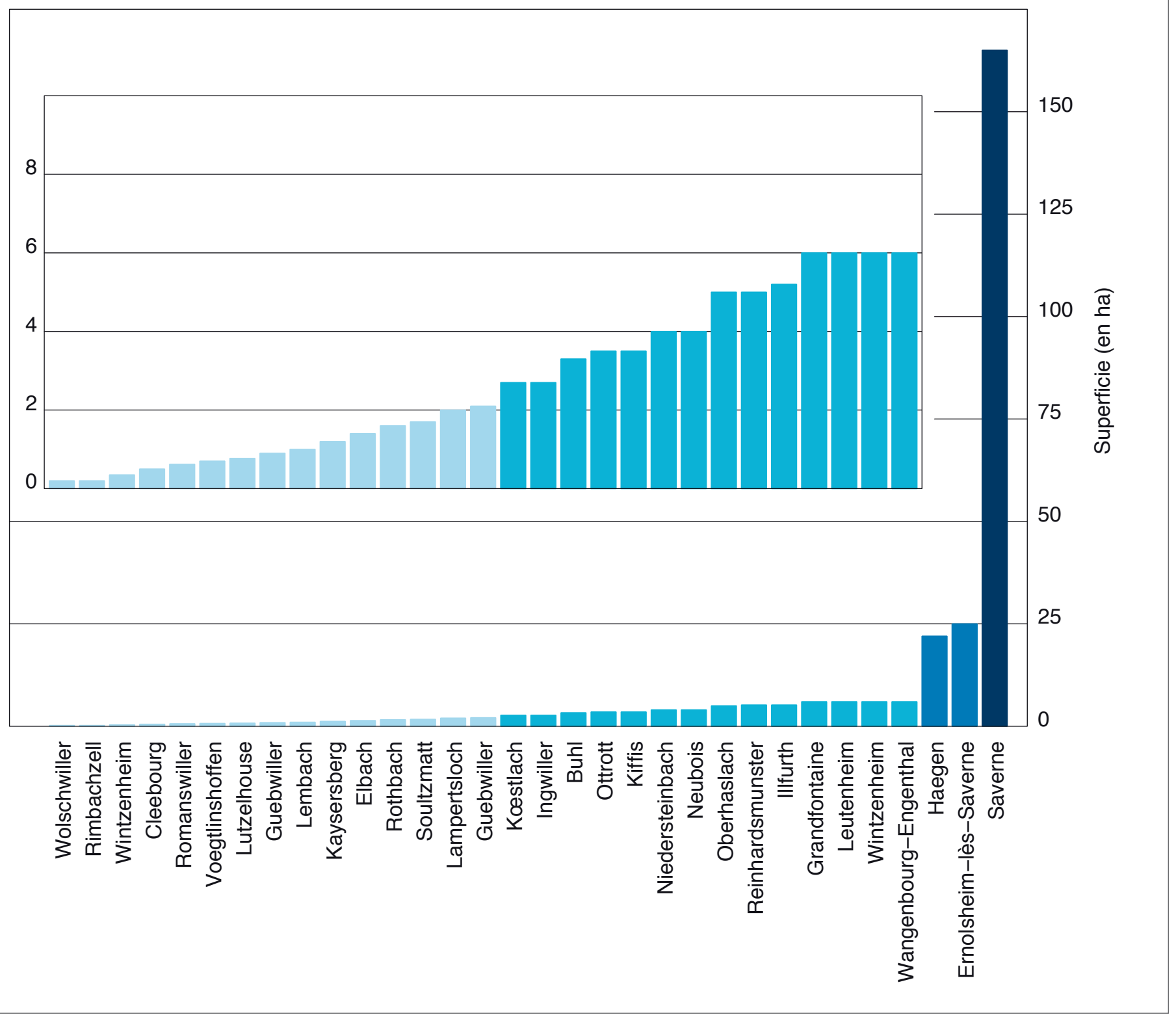

I3 Superficie des sites - DAO : C. Féliu. 
autres par leurs tailles importantes. Le premier, le Fossé des Pandours à Saverne, domine très largement l'ensemble avec sa surface de 165 ha; daté de La Tène finale, il s'intègre à la catégorie des oppida de la fin de l'âge du Fer, dont il représente l'un des exemplaires les plus importants pour la Gaule. Les deux autres, La Heidenstadt à Ernolsheim-lès-Saverne et le Brotschberg à Haegen, sont plus petits ; leur superficie s'établit respectivement à 25 et 22 ha. Tous trois sont situés à proximité du point le plus étroit du massif vosgien, le seuil de Saverne. La question du rôle des voies de communication importantes entre l'Alsace et le plateau lorrain dans la mise en place de sites de grande taille peut être posée.

Au sein de l'ensemble des plus petites fortifications, dont les superficies ne dépassent pas 8 ha, une rupture semble s'établir entre 2,1 et 2,6 ha et permet de définir deux groupes supplémentaires, dont les éléments constitutifs se répartissent assez régulièrement dans le territoire, avec toutefois une légère surreprésentation des sites les plus petits au nord de la zone d'étude et dans le sud du massif vosgien.

\subsection{Architecture des remparts}

Les recherches récentes menées sur les fortifications protohistoriques ont permis de renouveler les connaissances sur l'architecture des remparts. Plusieurs ouvrages ont été coupés et étudiés ; ils illustrent la grande variété des solutions architecturales mises en œuvre dans la vallée du Rhin supérieur, et en Alsace en particulier.

Au Hexenberg à Leutenheim, le site qui se développe au sommet d'une butte témoin à la fin de l'âge du Bronze final (BF IIIb) est fortifié par une palissade dont la présence a été reconnue dans chacun des sondages effectués au niveau de la rupture de pente. Cet aménagement, dont il ne reste que la tranchée de fondation, large d'une cinquantaine de centimètres et profonde de 40 à 70 centimètres, parfois dédoublée à l'occasion de réfections, était renforcé par un talus, actuellement presque totalement arasé, qui subsiste par endroit sur une dizaine de centimètres d'élévation (Lasserre et al. 2011).

Au Britzgyberg, les fouilles menées depuis une dizaine d'années ont permis de préciser l'architecture, déjà étudiée dans les années 1980, de deux lignes de fortification sur le flanc méridional du site. La première, la "palanque", correspond à une puissante palissade, implantée dans une tranchée de fondation large de $0,80 \mathrm{~m}$ en moyenne et profonde de 1,5 à 1,8 m, installée sur le rebord du plateau sommital. Ce dispositif était complété, à trois mètres en arrière, par une ligne de trous de poteau de grandes dimensions, les fondations des supports d'un portique interne qui devait ajouter à la monumentalité de l'ensemble. Celle-ci était encore soulignée par l'aménagement des pentes de la terrasse en un profond fossé. La construction de ce dispositif remonte au Hallstatt D1 et correspond à la première phase de fortification du site (Adam 2016). La seconde ligne de rempart étudiée récemment est située sur la terrasse inférieure et correspond à une période plus récente de l'occupation, datée de la fin du Hallstatt D2 ou du Hallstatt D3. Il adopte une architecture particulière, qui met en œuvre un front palissadé, retenant une masse de sédiment formant le corps du rempart, renforcé ou contreventé par un massif de pierres situé à l'avant de l'ouvrage (Adam 2010), selon un procédé peu courant.

La reprise de la fouille du Kastelberg à Kœstlach a permis de confirmer quelques observations effectuées au début du $\mathrm{XX}^{\mathrm{e}}$ s. par K. Gutmann. La fouille du rempart de barrage, le rempart 1 , montre une architecture très arasée, organisée autour d'un parement de petites dalles de calcaire, très mal conservé, qui retenait un talus de $8 \mathrm{~m}$ de large environ. La présence d'un unique trou de poteau dans le parement, situé en bordure de sondage, pose la question de la technique utilisée ou de la présence éventuelle d'un aménagement particulier qui mettrait en œuvre un support vertical. L'un des principaux résultats de la fouille correspond à la datation de la mise en place du rempart, au Hallstatt D1, voire peut-être même à la fin du Hallstatt $\mathrm{C}$. Une seconde ligne de fortification moins bien conservée encore, parallèle à la première sera ensuite installée, hypothétiquement à La Tène ancienne (Landolt et al. 2014 ; Landolt, Fleischer 2014).

Pour la fin de l'âge du Fer, les résultats sont plus nombreux. Au Fossé des Pandours, au-dessus de Saverne, la fouille du rempart de barrage, construit dans le courant de La Tène D1, a mis au jour les vestiges d'un murus gallicus, conservé sur une hauteur de $6,5 \mathrm{~m}$, pour une emprise au sol de 12 à $15 \mathrm{~m}$. Ce rempart, parfaitement rectiligne sur près de $600 \mathrm{~m}$ de long, est installé sur un terrain soigneusement préparé par la réalisation d'une marche horizontale qui entaille le terrain naturel sur $2 \mathrm{~m}$ de profondeur et qui accueillait les grilles de poutres situées dans la partie avant de l'architecture. Le parement est constitué de blocs soigneusement taillés (Fichtl 2010). Différents secteurs 
du rempart de contour, long de $7 \mathrm{~km}$, ont également été explorés : ils montrent des architectures moins soignées, parfois installées dans la pente, sans qu'aucun terrassement préparatoire n'ait été effectué avant la construction. On note toutefois que les parements sont toujours montés à l'aide de blocs taillés. Dans une phase de contraction de la zone enclose, un nouveau tronçon de rempart est construit au sud du site. Il ne correspond plus à un murus gallicus, mais à un édifice à poteaux frontaux, espacés de $4 \mathrm{~m}$ les uns des autres en moyenne. On assiste donc à la mise en place d'un programme de fortification mixte, où le rempart de barrage est armé de grilles de poutres horizontales et où certains segments du rempart de contour sont construits selon une architecture de type Pfostenschlitzmauer (Féliu 2010a).

À Ernolsheim-lès-Saverne, la Heidenstadt est fortifiée dans le courant de La Tène finale. Le rempart occidental a été coupé lors de deux campagnes de fouille en 2007 et 2008. L'architecture mise au jour est organisée autour de poteaux frontaux espacés de $2,5 \mathrm{~m}$ environ et liés entre eux par des poutres horizontales visibles dans le parement, dont près de la moitié des surfaces était composée de bois. Cette grille de madriers retenait la masse de matériaux qui formait le corps du rempart, dont les blocs de façade étaient pour certains taillés en diamant et ne pouvaient par conséquent pas tenir sans être arrimés à l'édifice (Féliu 2010b).

Au Frankenbourg, enfin, les fouilles en cours ont mis en évidence une architecture à poteaux verticaux et parements de blocs bruts à peine équarris, qui se distingue des autres remparts décrits ici par l'utilisation de pierres de très gros module dans le blocage interne de la construction (Féliu 2016).

Ces quelques lignes mettent en lumière la multiplicité des types architecturaux mis en œuvre dans la construction des remparts protohistoriques. Si l'importance de cette variabilité doit être soulignée à l'échelle régionale, elle est encore plus marquée quand elle s'exprime sur un site unique comme au Fossé des Pandours, où la cohérence du programme architectural, qui semblait pouvoir être mis en évidence dans un premier temps, s'estompe lors de la construction du rempart de réduction. Le nombre de fortifications à poteaux frontaux, généralement considérées comme caractéristiques du domaine oriental de l'espace nord-alpin à l'âge du Fer, doit également être souligné ; il ancre, comme d'autres marqueurs, l'Alsace aux régions d'Europe centrale.

\section{Analyse chronologique}

Le petit ensemble de fortifications retenues dans le cadre de cette étude peut être abordé par le prisme de la chronologie. Les trois principales périodes de mise en place des fortifications, l'âge du Bronze, le Hallstatt et La Tène ancienne et enfin La Tène finale, seront successivement envisagées (Fig. 4)

\subsection{L'âge du Bronze}

La documentation sur les fortifications de l'âge du Bronze est relativement peu étoffée. Seules deux occupations structurées, le Hohlandsberg (Fig. 5A) et le Hexenberg (Fig. 5B) appartiennent de façon assurée à ces périodes. Les différences marquées entre ces deux sites interdisent toutefois de tirer des conclusions trop définitives de leur confrontation.

Le Hohlandsberg occupe une position topographique avantageuse, au sommet d'une éminence qui domine la plaine du Rhin et le débouché de la vallée de la Fecht (Delrieu, Koch 2010). Il connaît une fréquentation sporadique à la fin du Néolithique ou au début de l'âge du Bronze et voit s'installer une première occupation d'importance à la fin du Bronze moyen ou au tout début du Bronze final (Bronze final I). Du mobilier de cette période a été découvert sur la quasi totalité de l'emprise que délimitera, à la phase suivante, l'enceinte. Quelques traces de bâtiments ont été mises au jour en bas de pente, à l'extrémité orientale de cette occupation qu'il est relativement difficile de caractériser, et dont le caractère non fortifié doit être souligné. Dans l'état actuel de nos connaissances sur le site, un hiatus, centré sur la phase ancienne du Bronze final, fait suite à cette première installation. Il sera suivi, entre le Bronze final II et le Bronze final IIIb qui correspond à l'abandon du site, par une nouvelle occupation. Celle-ci couvre environ 6 ha délimités par une enceinte dont le tracé implanté dans la pente, comme on a pu le voir plus haut, est atypique. Son architecture, qui n'a pas fait l'objet de recherches approfondies, n'est pas connue avec précision; elle est conservée sur $0,5 \mathrm{~m}$ d'élévation seulement et semble constituée d'un amoncellement de pierres. Les vestiges de bâtiments, appuyés sur cette enceinte ont été mis au jour dans le bas du site. Immédiatement à l'est du château médiéval qui couronne le site, un tronçon de système défensif a été découvert à la fin des 

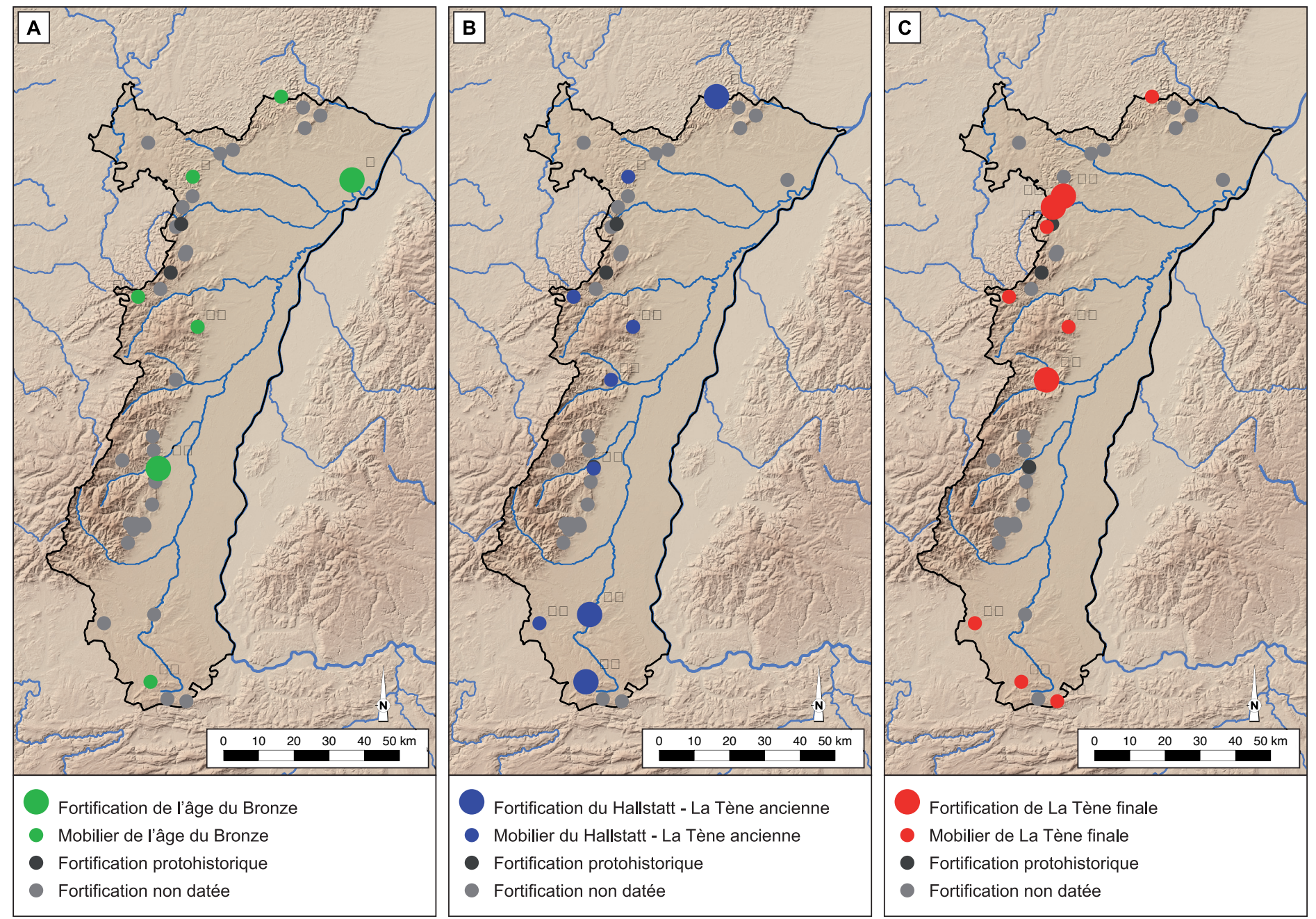

4 Localisation des fortifications de l'âge du Bronze (A), du Hallstatt-La Tène ancienne (B) et de La Tène finale (C) DAO : C. Féliu (fond : SRTM-NASA).

années 1990. Il pourrait correspondre à une délimitation de la partie sommitale de l'occupation, où un four de potier et de très nombreux ratés de cuisson ont été découverts.

La butte du Hexenberg à Leutenheim est située au nord de l'Alsace, dans la plaine du Rhin dont elle domine les méandres et les bras morts d'une dizaine de mètres (Adam, Lasserre 2001 ; Lasserre et al. 2011). Elle est occupée de façon assez brève à la toute fin de l'âge du Bronze final (Bronze final IIIb). Un nombre important de structures en creux a été mis au jour dans les sondages ouverts sur le site. Il laisse envisager une occupation relativement dense à l'intérieur de la palissade, sur le sommet plat de la butte.

D'autres sites ont livrés les traces fugaces d'une occupation plus ou moins importante durant l'âge du Bronze. Le rocher du Mont Sainte-Odile a livré du mobilier du Bronze moyen et des phases anciennes et moyenne du Bronze final (Zumstein 1993); au Maimont à Niedersteinbach, du mobilier est mentionné, comme au Donon ou à Dossenheim-sur-Zinsel. À Kœstlach, quelques rares objets et une datation radiocarbone effectuée sur un os découvert en position secondaire dans une couche de démolition du rempart de l'âge du Fer, permettent de proposer une occupation, ou une fréquentation, du site entre le Bronze moyen et le début du Bronze final (Landolt, Fleischer 2014).

L'ensemble de ces indices inscrit les fortifications alsaciennes de l'âge du Bronze dans une dynamique plus large qui voit ce type d'installation se développer dans toute la France non méditerranéenne. On notera, à l'échelle de la région, que les surfaces encloses restent relativement 
modestes et ne dépassent pas 6 ha. D'un point de vue chronologique, les occupations du Bronze moyen et du début du Bronze final (Hohlandsberg, Ernolsheim-lès-Saverne, Mont Sainte-Odile) sont assez peu nombreuses et méritent d'être soulignées. Enfin, il paraît difficile, dans l'immédiat, de proposer un modèle synthétique pour les fortifications de l'âge du Bronze en Alsace tant les sites concernés semblent recouvrir des réalités différentes.

\subsection{Le premier âge du Fer et le début du second}

La documentation relative aux fortifications de l'âge du Fer en général est un peu plus abondante que pour la période précédente, avec près d'une quinzaine de sites concernés. Pour le Hallstatt et La Tène ancienne, les deux ensembles pour lesquelles les données sont les plus nombreuses et les plus fiables sont le Kastelberg à Kœstlach (Fig. 5C) et le Britzgyberg à Illfurth (Fig. 5D)

Le premier occupe l'extrémité d'un éperon, déjà fortifié au Néolithique et défendu, à l'âge du Fer par un rempart de barrage éventuellement prolongé par un rempart de contour qui enserre une superficie de 2,6 ha (Landolt et al. 2014 ; Landolt, Fleischer 2014). Les sondages, qui se sont concentrés sur le système défensif n'ont pas mis en évidence de structures liées à l'occupation interne. La reprise du mobilier de fouilles anciennes permet néanmoins de proposer une occupation centrée sur le Hallstatt D1, qui débuterait peut-être même au Hallstatt C ; les fouilles récentes permettent de dater de la même façon la mise en place de la fortification.

À une vingtaine de kilomètres au nord, le site du Britzgyberg est installé sur un éperon qui domine la vallée de 1'Ill. Sa topographie est marquée par un système de deux terrasses emboitées, la plus basse entourant la seconde sur trois côtés. Le site est bien connu par des fouilles réalisées depuis les années 1960 ; il est intégré de longue date à la réflexion sur les "résidences princières" du Hallstatt final (Schweitzer 1997, par exemple). La découverte d'un mobilier abondant, caractérisé par de nombreuses importations, dont de la céramique attique et des amphores massaliètes, trahit l'importance du site. Une activité métallurgique du bronze et du fer doit également être mentionnée. Outre les avancées sur les fortifications, dont il a déjà été question plus haut, les recherches récentes ont permis de préciser la chronologie et l'évolution des occupations du site entre le Hallstatt D1 et La Tène ancienne (Adam 2010).
Un troisième site, dont la reprise de la fouille a démarré en 2016, a livré les vestiges d'une fortification de la fin du Hallstatt ou de La Tène ancienne. Le Maimont à Niedersteinbach est situé à l'extrême nord de l'Alsace, à cheval sur la frontière avec le Palatinat et l'Allemagne. Installé sur un sommet qui surplombe une petite vallée secondaire, cette petite fortification de 4 ha était protégée par un système de remparts successifs qui ont fait l'objet d'une coupe en 1996. Plusieurs états de construction ont été reconnus. Une date radiocarbone permet de proposer que le premier d'entre eux puisse dater du Hallstatt final ou de La Tène ancienne (Fichtl, Adam 2001). Elle constitue le seul indice d'une occupation du site à la fin du premier ou au début du second âge du Fer ; le mobilier découvert anciennement serait plutôt daté de La Tène finale et de l'époque romaine.

D'autres sites enfin ont également livré du mobilier de cette période. Au Mont Sainte-Odile, de la céramique de La Tène ancienne a été mise au jour lors des fouilles sur le rocher du couvent, tout comme au sommet du Donon. Au Frankenbourg, à Neubois, du mobilier métallique a été découvert lors de prospections : une pendeloque panier provenant de la culture de Golaseca et un fragment de fibule à timbale permettent de proposer une fréquentation du site au Hallstatt final ; quatre fibules de La Tène ancienne viennent compléter ce petit ensemble (Féliu, Tremblay Cormier 2014 ; Féliu 2018). Quelques tessons du Hallstatt ont été mis au jour lors des fouilles menées depuis, mais aucune structure de cette période n'a jusqu'à maintenant été découverte.

On note, un fois encore, que l'Alsace s'inscrit dans un mouvement plus général de développement des fortifications à la fin du premier et au début du second âge du Fer. Les deux phénomènes complémentaires observés au nord des Alpes sont illustrés sur la rive gauche du Rhin : on assiste d'une part à la multiplication, dans le courant du premier âge du Fer, des habitats fortifiés de hauteur, généralement de petite taille, et d'autre part, au développement, au Hallstatt final du phénomène des sites "princiers". Bien que cette dernière catégorie ne soit plus opérante, il n'en reste pas moins que certains sites, comme le Britzgyberg, semblent polariser les échanges économiques à cette époque. L'occupation du Frankenbourg, pourrait éventuellement représenter une catégorie intermédiaire au sein des habitats de hauteur du premier âge du Fer de la région du Rhin supérieur. 

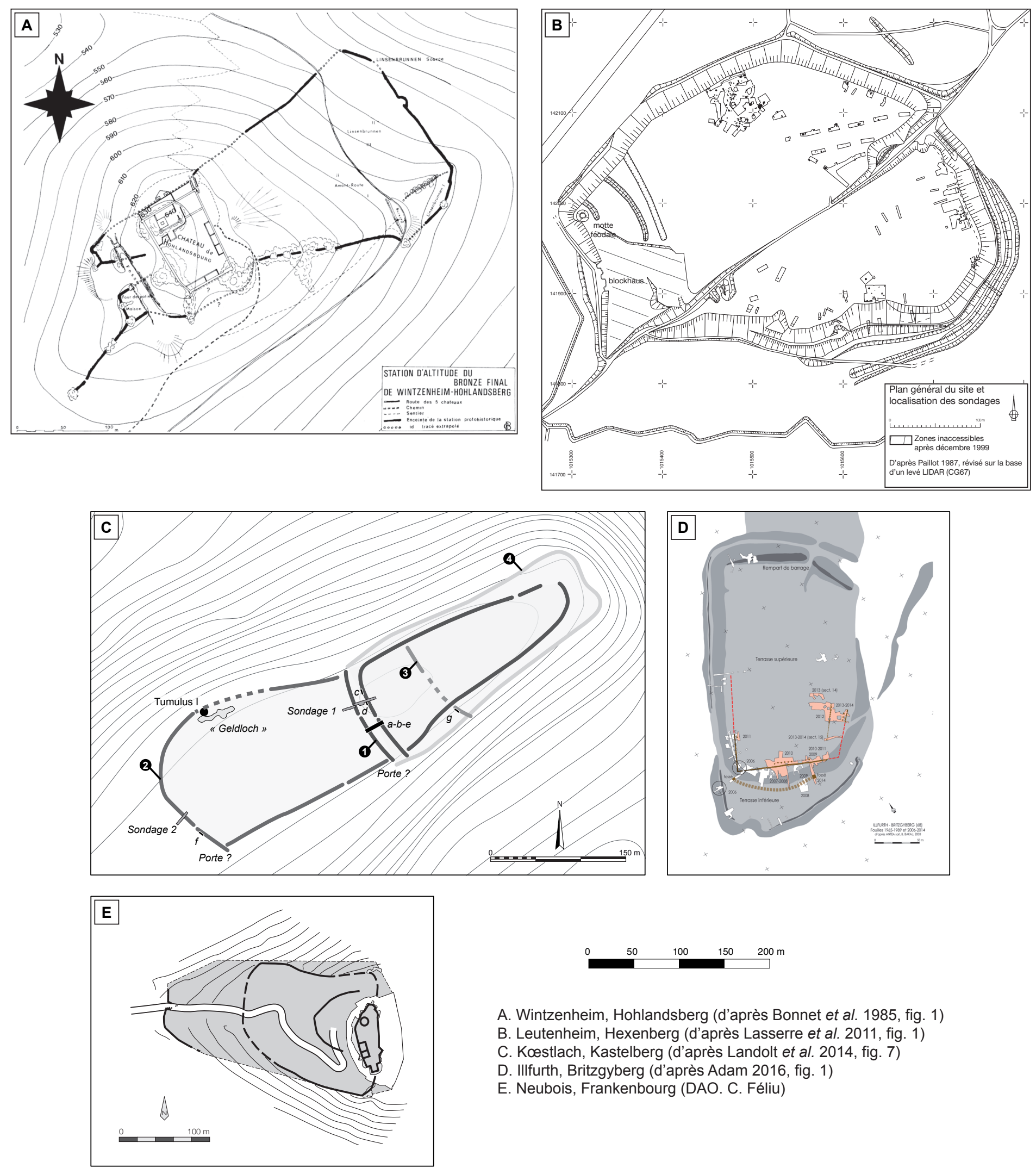

A. Wintzenheim, Hohlandsberg (d'après Bonnet et al. 1985, fig. 1)

B. Leutenheim, Hexenberg (d'après Lasserre et al. 2011, fig. 1)

C. Kœstlach, Kastelberg (d'après Landolt et al. 2014, fig. 7)

D. Illfurth, Britzgyberg (d'après Adam 2016, fig. 1)

E. Neubois, Frankenbourg (DAO. C. Féliu)

15 Plans d'une sélection de sites fortifiés (échelle 1/6000). 


\subsection{La Tène finale}

Pour la fin de l'âge du Fer trois sites seulement ont livré les vestiges structurés d'une occupation fortifiée. Le plus important d'entre eux est l'oppidum du Fossé des Pandours, implanté sur la voie de passage la plus aisée des Vosges, au Col de Saverne. Sa superficie nettement plus importante que celle des autres sites du même type dans la région (165 ha), l'importance des activités économiques qui y sont attestées (importation, artisanat) comme la découverte des vestiges mobiliers d'un atelier monétaire permettent de le considérer comme la capitale de la cité des Médiomatriques à la fin du $\mathrm{II}^{\mathrm{e}}$ et au début du $\mathrm{I}^{\mathrm{er}} \mathrm{s}$. av. J.-C. (Fichtl 2003). À quelques kilomètres au nord, la Heidenstadt est également un oppidum, beaucoup plus petit avec 25 ha seulement de superficie, qui peut être daté, sur la base d'un mobilier très peu abondant, d'une phase un peu plus récente de La Tène finale. Son installation pourrait être liée au déplacement du centre de gravité de la cité des Médiomatriques vers Metz et à l'abandon du Fossé des Pandours dans le courant du Ir s. av. J.-C. Elle doit également être mise en relation avec le contrôle du point de passage que constituait le seuil de Saverne (Féliu 2011).

Au Frankenbourg (Fig. 5E), la construction du rempart inférieur et de la porte qui le perçait peut être datée de La Tène finale. Elle correspond à la phase protohistorique la mieux représentée dans le mobilier mis au jour lors des prospections et des fouilles : plus d'une dizaine de fibules, une quarantaine de monnaies, des fragments de vaisselle en bronze et de nombreux tessons d'amphores appartiennent à cette phase de l'occupation et indiquent son importance. La découverte de nombreuses scories de fer dans les niveaux de la fin de l'âge du Fer témoigne de la présence sur le site d'une activité métallurgique assez importante et vient renforcer cette image (Féliu 2018).

Les autres sites pour lesquels on peut proposer une occupation ou un fréquentation à La Tène finale n'ont généralement livré que du mobilier de cette période. Au Maimont, de la céramique et un éventuel "fond de cabane" sont mentionnés au début du $X^{\mathrm{e}} \mathrm{s}$. À Reinhardtmunster, comme à Elbach, Kœstlach ou Wolschwiller, des découvertes plus ou moins anciennes et bien documentées permettent d'évoquer l'hypothèse d'occupations à la fin de l'âge du Fer. Enfin, les fouilles du rocher du couvent ont livré un abondant mobilier de La Tène finale qui incite à restituer un site d'importance à l'emplacement du monastère actuel qui en a toutefois fait disparaître toutes structures (Châtelet, Baudoux 2015).

Ces quelques sites montrent les changements à l'œuvre dans les modalités d'occupation des sites fortifiés à la Tène finale : si cette forme d'habitat refait son apparition dans les paysages de la fin de l'âge du Fer, les sites les plus importants couvrent maintenant de grandes superficies, alors qu'une série de petits établissements de hauteur sont créés ou réoccupés.

\section{Conclusions, perspectives}

Les quelques points exposés plus haut permettent de tirer les enseignements des études nouvelles sur les fortifications et les enceintes de hauteur alsaciennes. Tout d'abord, on peut noter que l'un des domaines pour lesquels les résultats sont les plus immédiatement accessibles concerne l'architecture des remparts. En effet, pas moins de huit de ces monuments ont été coupés et étudiés, permettant ainsi de préciser les typologies et de nuancer la césure traditionnellement établie sur le Rhin entre rempart à poutrage horizontal et construction de type Pfostenschlitzmauer.

Si elle ne permet que d'approcher le détail de l'organisation interne de ces sites qui résiste encore à l'analyse, la multiplication des fouilles offre toutefois l'opportunité de réviser, approfondir ou établir la datation des enceintes. Les grandes évolutions chronologiques visibles en Alsace correspondent dans les grandes lignes à celles que l'on peut observer dans les régions voisines et plus largement à l'échelle de l'Europe, avec des phases de développement de ce type d'occupation importantes, âge du Bronze final, Hallstatt final - La Tène ancienne ou La Tène finale, entrecoupées de périodes de déprise et d'abandon des hauteurs. On notera toutefois que les quelques occupations de la fin du Bronze moyen et du début du Bronze final, au Hohlandsberg, au Mont Sainte-Odile ou à Kœstlach, font figure de cas particulier.

La multiplication des datations et leur précision accrue incitent à considérer les fortifications sur un temps long, qui dépasse les cadres habituels du découpage chronologique traditionnel. En effet, on note, dans l'évolution globale de ces sites, que certains d'entre eux connaissent de nombreuses phases d'utilisations qui peuvent commencer dès le Néolithique et se poursuivent généralement au 
Bas-Empire et éventuellement durant tout le Moyen Âge, alors que d'autres ne connaissent qu'une courte occupation. Ces différences ne semblent pas devoir s'expliquer par la localisation ou la position stratégique des sites en question : si le Frankenbourg, qui est situé sur une voie de franchissement des Vosges est occupé à de très nombreuses reprises entre le Hallstatt final et le Moyen Âge, c'est aussi le cas du Maimont, qui se trouve, pour sa part légèrement à l'écart, dans une vallée secondaire. Au contraire, le Fossé des Pandours, dont la position sur le Col de Saverne ne saurait être plus avantageuse, n'est pleinement développé que durant un temps relativement court, entre la fin du $\mathrm{II}^{\mathrm{e}}$ et le milieu du I ${ }^{\text {er }}$ s. av. J.-C. D'autres facteurs explicatifs, peut-être les ressources naturelles, devront être interrogés pour réintégrer les enceintes dans leur contexte général et comprendre leurs spécificités.

De même que les bornes chronologiques de l'étude de l'habitat fortifié doivent être écartées, le cadre géographique nécessite d'être élargi pour une meilleure compréhension des évolutions de l'habitat fortifié protohistorique. Il est en effet peu pertinent de ne pas prendre en compte les fortifications du pendant allemand des fortifications vosgiennes pour saisir leur fonctionnement qui semble, pour certaines, lié aux axes de circulation, dont la plaine du Rhin dans son ensemble faisait partie. Il semble difficile d'appréhender l'importance de sites comme le Britzgyberg ou le Frankenbourg au Hallstatt, par exemple, sans les confronter à celui du Münsterberg de Breisach. De même, ne pas intégrer les enceintes du versant occidental des Vosges ne permet pas d'envisager pleinement le rôle des fortifications alsaciennes dans ce contexte particulier (Walter 2016).

Enfin, les travaux récents l'ont montré, d'une façon encore morcelée qui mériterait d'être systématisée, l'utilisation de données dont la définition géographique est relativement large, comme le Lidar disponible pour l'ensemble du territoire du Haut-Rhin (Wittlin 2016), ou l'interrogation de critères liés à l'environnement des sites aisément manipulables par le biais de SIG, comme les relations d'inter-visibilité (Robin 2016) ou les analyses de distance-coût (Walter 2015) offrent une palette d'outils et de marqueurs, déjà anciennement utilisés par d'autres, mais propre à renouveler les approches et les interprétations des sites fortifiés. Le court bilan proposé ici ne se veut pas être un bilan définitif, mais plutôt un point d'étape pour préparer de nouveaux développements.

\section{Clément FÉliu}

Inrap / UMR 7044 - Archimède

10, rue d'Altkirch - 67100 Strasbourg clement.feliu@inrap.fr

Références bibliographiques

Adam 2010 : ADAM (A.-M.) - Genese und Entwicklung der befestigten Höhensiedlung auf dem Britzgyberg in Illfurth (Haut-Rhin, Frankreich). In : KRAUSSE (D.) dir. - «Fürstensitze » und Zentralorte der frühen Kelten : Abschlusskolloquium des DFG-Schwerpunktprogramms 1171 in Stuttgart, 12.-15.Oktober 2009. Stuttgart, Theiss, 2010, p. 365-375

Adam 2016 : ADAM (A.-M.) - La palissade dans tous ses états : l'enclos du Britzgyberg (Illfurth, Haut-Rhin) et autres aménagements palissadés dans les habitats du premier âge du Fer. In : FÉLIU (C.), SCHWIEN (J.-J.) dir. - Des fossés et des remparts ; enceintes et sites fortifiés du Rhin Supérieur entre Protohistoire et Moyen Âge : dossier thématique. Archimède, 3, 2016. [en ligne : http://archimede.unistra.fr/revue-archimede/archimede-3-2016/ archimede-3-2016-dossier-la-palissade-dans-tous-ses-etats/].

Adam, Lasserre 2001 : ADAM (A.-M.), LASSERRE (M.) - La butte de Hexenberg à Leutenheim (Bas-Rhin) : un habitat fortifié de la fin du Bronze final, fouilles 1994 à 1999. Bulletin de la Société archéologique de France, 98-2, 2001, p. 311-324.

Bleicher, Faudel 1880 : BLEICHER (G.), FAUDEL (F.) - Matériaux pour une étude préhistorique de l'Alsace; monuments mégalithiques, deuxième partie. Bulletin de la Société d'histoire naturelle de Colmar, 21, 1880, p. 83-183.
Bonnet et al. 1985 : BONNET (C.), PLOUIN (S.), LAMBACH (F.) - Linsenbrunnen II, un nouveau secteur de la station d'altitude de Hohlandsberg (commune de Wintzenheim, Haut-Rhin). Bulletin de la Société préhistorique française, 82-10, 1985, p. 449-509.

Buchsenschutz 1984 : BUCHSENSCHUTZ (O.) - Structures d'habitat et fortifications de l'âge du Fer en France septentrionale. Paris, Société préhistorique française, 1984, 247 p. (Mémoires de la Société Préhistorique Française, 18).

Châtelet, Baudoux 2015 : CHÂTELET (M.), BAUDOUX (J.) - Le « mur païen » du Mont Sainte-Odile en Alsace, un ouvrage du haut Moyen Âge ? L'apport des fouilles archéologiques. Zeitschrift für Archäologie des Mittelalters, 43, 2015, p. 1-27.

Delrieu, Koch 2010 : DELRIEU (F.), KOCH (J.) dir. - Le massif du Hohlandsberg, Haut-Rhin ; archéologie d'un espace naturel homogène : rapport de PCR. Sélestat, PAIR, 2010. 2 volumes.

Deyber 1984 : DEYBER (A.) - structures et fonctions des fortifications de l'âge du Fer dans le nord-est de la France (régions Champagne-Lorraine-Alsace). In : CAHEN-DELHAYE (A.), DUVAL (A.), LEMAN-DELERIVE (G.), LEMAN (P.) dir. - Les Celtes en Belgique et dans le nord de la France ; les fortifications de l'Age du Fer: actes du $6^{\mathrm{e}}$ colloque de l'AFEAF, Bavay et Mons, 1982. Villeneuve d'Ascq, Revue du Nord, 1984, p. 215-236 (Revue $d u$ Nord, numéro spécial hors série). 
Féliu 2010a : FÉLIU (C.) - Quelques observations architecturales sur les remparts de contour de l'oppidum du Fossé des Pandours au Col de Saverne (Bas-Rhin). In : FICHTL (S.) dir. - Murus celticus ; architecture et fonctions des remparts de l'âge du Fer. Actes de la table ronde internationale de Bibracte, 11-12 octobre 2006. Glux-en-Glenne, Bibracte, 2010, p. 77-85.

Féliu 2010b : FÉLIU (C.) - Le rempart de la Heidenstadt à Ernolsheim-lès-Saverne (Bas-Rhin). In : FICHTL (S.) dir. - Murus celticus ; architecture et fonctions des remparts de l'âge du Fer. Actes de la table ronde internationale de Bibracte, 11-12 octobre 2006. Glux-en-Glenne, Bibracte, 2010, p. 211-221.

Féliu 2011 : FÉLIU (C.) - L'oppidum de la Heidenstadt et le seuil de Saverne à la fin de l'âge du Fer. Cahiers Alsaciens d'Archéologie d'Art et d'Histoire, 54, 2011, p.47-64.

Féliu 2016 : FÉLIU (C.) - L'enceinte inférieure du Frankenbourg (67) et les remparts à poteaux frontaux de la fin de l'âge du Fer dans l'espace du Rhin supérieur. Pour une révision de la typologie des Pfostenschlitzmauern. In : FÉLIU (C.), SCHWIEN (J.-J.) dir. - Des fossés et des remparts ; enceintes et sites fortifiés du Rhin Supérieur entre Protohistoire et Moyen Âge : dossier thématique. Archimède, 3, 2016. [en ligne : http://archimede.unistra. fr/revue-archimede/archimede-3-2016/archimede-3-2016-dossier-lenceinte-inferieure-du-frankenbourg/].

Féliu 2018 : FÉLIU (C.) dir. - La fortification du Frankenbourg à Neubois (Bas-Rhin) : rapport triennal de fouille programmée. Strasbourg, Metz : Inrap, SRA Alsace, 2018

Féliu, Schwien 2016 : FÉLIU (C.), SCHWIEN (J.-J.) dir. - Des fossés et des remparts ; enceintes et sites fortifiés du Rhin Supérieur entre Protohistoire et Moyen Âge : dossier thématique. Archimède, 3, 2016. [en ligne : http:// archimede.unistra.fr/revue-archimede/archimede-3-2016/\#c79404].

Féliu, Tremblay Cormier 2014 : FÉLIU (C.), TREMBLAY CORMIER (L.) - Indices d'une occupation hallstattienne au Frankenbourg (Neubois, Bas-Rhin). In : ALBERTI (G.), FÉLIU (C.), PIERREVELCIN (G.) dir. Transalpinare, mélanges offerts à Anne-Marie Adam. Bordeaux, Ausonius, 2014, p. 229-234.

Fichtl 2003 : FICHTL (S.) - L'oppidum du fossé des Pandours, chef-lieu des Médiomatriques à l'époque de 1'Indépendance ? Archaeologia Mosellana, 5, 2003, p. 145-160.

Fichtl 2010 : FICHTL (S.) - L'architecture murus gallicus de 1'oppidum du Fossé des Pandours au Col de Saverne (Bas-Rhin). In : FICHTL (S.) dir. - Murus celticus ; architecture et fonctions des remparts de l'âge du Fer. Actes de la table ronde internationale de Bibracte, 11-12 octobre 2006. Glux-en-Glenne, Bibracte, 2010, p. 85-91.

Fichtl, Adam, Morand 1997 : FICHTL (S.), ADAM (A.-M), MORAND (M.-J.) - Recherches actuelles sur les enceintes de Basse-Alsace de la Protohistoire au Bas-Empire. Revue d'Alsace, 123, 1997, p. 3-18.

Fichtl, Adam 2001 : FICHTL (S.), ADAM (A.-M.) - Le Maimont, près de Niedersteinbach, une enceinte fortifiée dans les Vosges du Nord. Cahiers alsaciens d'archéologie, d'art et d'histoire, 44, 2001, p. 5-12.

Flotté, Fuchs 2000 : FLOTTÉ (P.), FUCHS (M.) - Carte archéologique de la Gaule, le Bas-Rhin, 67/1. Paris, Académie des Inscriptions et Belles-Lettres, $735 \mathrm{p}$.

Forrer 1926 : FORRER (R.) - Des enceintes fortifiées préhistoriques, romaines et anhistoriques d'Alsace. Bulletin de la Société pour la conservation des monuments historiques d'Alsace, $\mathrm{II}^{\mathrm{e}}$ série, 26, 1926, p. 1-73.

Gutmann 1913 : GUTMANN (K.-S.) - Die neolitische Bergfeste von Oltingen. Praehistorische Zeitschrift, 5, 1913, p. 158-205.
Landolt, Fleischer, Putelat 2014 : LANDOLT (M.), FLEISCHER (F.), PUTELAT (O.) - Nouveaux éléments sur la chronologie du site de hauteur fortifié pré- et protohistorique du "Kastelberg" à Kœstlach-Mœrnach (HautRhin). In : ALBERTI (G.), FÉLIU (C.), PIERREVELCIN (G.) dir. - Transalpinare, mélanges offerts à Anne-Marie Adam. Bordeaux, Ausonius, 2014, p. 205-227.

Landolt, Fleischer 2014 : LANDOLT (M.), FLEISCHER (F.) dir. - Koestlach-Mornach, Haut-Rhin, "Kastelberg”, un site fortifié pré-et protohistorique sur les premiers contreforts jurassien : rapport de fouille programmée. Sélestat, PAIR, 2014, 175 p.

Lasserre et al. 2011 : LASSERRE (M.), VIGREUX (T.), BASOGE (F.), LOGEL (T.), PUTELAT (O.), SCHNEIDER (N.), MICHLER (M.), JODRY (F.) BOËS (X.) - Le site de la fin du Bronze final du Hexenberg à Leutenheim (Bas-Rhin) : études sur le paléoenvironnement rhénan et études archéologiques ; résultats préliminaires. Bulletin de la Société préhistorique française, 2011, 108-4, p. 731-754.

Mehlis 1900 : MEHLIS (C.) - Über vorgeschichtliche Befestigungen in den Nordvogesen und im Hartgebirge. Korrespondenzblatt des Gesammtvereins der deutschen Geschichts- und Altertumsvereine, 1900, p. 11-16.

Robin 2016 : ROBIN (A.) - Les fortifications du premier âge du Fer dans l'est de la Gaule ; fonction et organisation du territoire à la fin du premier âge du Fer (Ha D2/D3 - LT A) : mémoire de master. Strasbourg, Université de Strasbourg, 2016, $231 \mathrm{p}$.

Roussel-Claerr 1983 : ROUSSEL-CLAERR (C.) - Les habitats fortifiés pendant la pré-et protohistoire en Alsace, Franche-Comté et Lorraine : thèse de doctorat. Besançon, Université de Franche-Comté, 1983, 2 volumes.

Schoepflin, Ravenez 1849-1852 : SCHOEPFLIN (J.-D.), traduit par RAVENEZ (L. W.) - L'Alsace illustrée, ou recherches sur l'Alsace pendant la domination des Celtes, des Romains, des Francs, des Allemands et des Français. Mulhouse, François Perrin, 1849, 1849, 1851, 1851, 1852, 5 volumes.

Schweitzer 1997 : SCHWEITZER (J.) - L'oppidum du Britzgyberg et le faciès hallstattien dans le horst de Mulhouse. In : BRUN (P.), CHAUME (B.) dir. Vix et les éphémères principautés celtiques ; les $V I^{e}-V^{e}$ siècles avant J.-C. en Europe centre-occidentale. Actes du colloque de Châtillon-sur-Seine, 27-29 octobre 1993. Paris, Errance, 1997, p. 57-66.

Walter 2015 : WALTER (M.) - Les sites fortifiés de hauteur du massif vosgien , actualisation des données et modalités d'implantation : mémoire de master. Strasbourg, Université de Strasbourg, 2015, 2 volumes.

Walter 2016 : WALTER (M.) - Les sites de hauteur du massif vosgien. Actualisation des données et modalités d'implantation (Néolithique récent $-\mathrm{X}^{\mathrm{e}}$ siècle). In : FÉLIU (C.), SCHWIEN (J.-J.) dir. - Des fossés et des remparts ; enceintes et sites fortifiés du Rhin Supérieur entre Protohistoire et Moyen Âge : dossier thématique. Archimède, 3, 2016. [en ligne : http://archimede. unistra.fr/revue-archimede/archimede-3-2016/archimede-3-2016-dossierles-sites-de-hauteur-du-massif-vosgien/].

Wittlin 2016 : WITTLIN (E.) - Etude diachronique des enceintes de hauteur du Haut-Rhin ; réactualisation des données anciennes sur les enceintes anhistoriques et nouvelles approches grâce aux données récentes, principalement l'imagerie LIDAR : mémoire de master. Strasbourg, Université de Strasbourg, 2016, 2 volumes.

Zehner 1998 : ZEHNER (M.) - Carte archéologique de la Gaule ; le HautRhin, 68. Paris, Académie des Inscriptions et Belles-Lettres, 1998, 374 p.

Zumstein 1993 : ZUMSTEIN (H.) - Deux campagnes de fouilles au Mont Sainte-Odile en 1965. Cahiers alsaciens d'archéologie d'art et d'histoire, 36,1993 , p. 89-95. 